\title{
First-Order Reorientation Transition of the Flux-Line Lattice in CaAlSi
}

\author{
P. K. Biswas, ${ }^{1, *}$ M. R. Lees, ${ }^{1}$ G. Balakrishnan, ${ }^{1}$ D. Q. Liao, ${ }^{1}$ D. S. Keeble, ${ }^{1}$ J. L. Gavilano, ${ }^{2}$ N. Egetenmeyer, ${ }^{2}$ \\ C. D. Dewhurst, ${ }^{3}$ and D. McK. Paul ${ }^{1}$ \\ ${ }^{1}$ Physics Department, University of Warwick, Coventry, CV4 7AL, United Kingdom \\ ${ }^{2}$ Paul Scherrer Institut, CH-5232 Villigen PSI, Switzerland \\ ${ }^{3}$ Institut Laue-Langevin, 6 Rue Jules Horowitz, F-38042 Grenoble, France
}

(Received 25 July 2011; published 13 February 2012)

\begin{abstract}
The flux-line lattice in CaAlSi has been studied by small-angle neutron scattering. A well-defined hexagonal flux-line lattice is seen just above $H_{c 1}$ in an applied field of only $54 \mathrm{Oe}$. A $30^{\circ}$ reorientation of this vortex lattice has been observed in a very low field of 200 Oe. This reorientation transition appears to be first-order and could be explained by nonlocal effects. The magnetic field dependence of the form factor is well-described by a single penetration depth of $\lambda=1496(1) \AA$ and a single coherence length of $\xi=307(1) \AA$ at $2 \mathrm{~K}$. At $1.5 \mathrm{~K}$, the penetration depth anisotropy is $\gamma_{\lambda}=2.7(1)$, with the field applied perpendicular to the $c$ axis, and agrees with the coherence length anisotropy determined from critical field measurements.
\end{abstract}

DOI: 10.1103/PhysRevLett.108.077001

The ternary silicide superconductor CaAlSi (CAS) [1-4] has the same $\mathrm{AlB}_{2}$-type layered structure as $\mathrm{MgB}_{2}$ [5]. CaAlSi exhibits a number of interesting superconducting properties, the study of which can provide an insight into the factors leading to high superconducting transition temperatures $\left(T_{c}\right)$ in this class of materials.

Neutron and x-ray diffraction studies have shown that there are two possible arrangements for the atoms in the AlSi layers in CaAlSi. These layers stack along the $c$ axis in a sequence $(A A B B B)$ in fivefold $5 H$-CAS and $(A A A B B B)$ in sixfold $6 H$-CAS. Further distortions produce either corrugated or flat AlSi layers within the multistack structures [4]. An unmodulated phase ( $1 \mathrm{H}$-CAS) can be grown by controlled cooling from the molten state [6].

The superconducting properties of CAS, including $H_{c 2}(T)$ and $T_{c}$ (5.7 to $7.8 \mathrm{~K}$ ), change with modulation, as does the anisotropy in the superconducting parameters $\gamma_{H}=H_{c 2}^{a b} / H_{c 2}^{c}$ and $\gamma_{\lambda}=\lambda_{c} / \lambda_{a b}$, although $\gamma$ values of 2-3 indicate that these materials are only moderately anisotropic [7-9]. The heat capacity of $6 \mathrm{H}$-CAS below $T_{c}$ is well-explained within the framework of the BCS theory with strong coupling, with a single superconducting gap, $2 \Delta$, at $T=0 \mathrm{~K}$ giving $2 \Delta / k_{B} T_{c}=4.07$ [3]. While the $T_{c}$ of $1 H$-CAS decreases with applied pressure $P, d T_{c} / d P=$ $+0.21 \mathrm{~K} / \mathrm{GPa}$ for $6 H$-CAS $[3,10]$.

The electronic structure of CAS consists of $\sigma$ and $\pi$ bands derived from hybridized $(\mathrm{Al}, \mathrm{Si}) s$ and $p$ states and Ca $s, p$, and $d$ states [11-13]. In 6H-CAS, calculations show that there are two disconnected cylindrical Fermi surfaces which have two-dimensional character [14]. Different measurement techniques suggest different scenarios for the superconducting gap. Angle-resolved photoemission spectroscopy measurements indicate that, in $6 \mathrm{H}$-CAS, there are two superconducting gaps with equal magnitudes [15]. Muon spin rotation studies of the field
PACS numbers: 74.25.Ha, 74.25.Uv, 74.70.Dd, 75.70.Kw

dependence of penetration depth $\lambda$ [9] and optical measurements both suggest an anisotropic or multigapped structure [16]. In contrast, tunnel-diode resonator measurements and break-junction tunneling spectroscopy both point to a single weakly anisotropic $s$-wave gap in $6 H$-CAS $[17,18]$.

In this Letter, we report the results of a small-angle neutron scattering (SANS) study of the magnetic fluxline lattice (FLL) in the $6 H$ phase of CAS. SANS is a powerful technique for studying the mixed state of type-II superconductors [19] and has often been used to investigate the symmetry of the pairing mechanism and the macroscopic physics of the FLL [20-27]. We demonstrate that the FLL in CAS has a reorientation transition at a relatively small applied magnetic field, similar to that seen in $\mathrm{MgB}_{2}$ at much higher fields [28]. This low-field transition is probably due to nonlocal effects, rather than multiband physics as suggested for $\mathrm{MgB}_{2}$. Our measurement of the field-dependent form factor from the field distribution is explained by a single coherence length, and the anisotropy of this coherence length is the same as the anisotropy of the penetration depth. Both features are very unlikely to occur in a multiband superconductor.

Single crystals of $6 H$-CAS were prepared by the Bridgman method [29]. Polycrystalline ingots of CAS were made by melting stoichiometric mixtures of calcium shot $(99.99 \%)$, aluminum shot $(99.999 \%)$, and silicon pieces $(99.99 \%)$ in an arc furnace under flowing argon gas. Each as cast ingot was placed in a boron nitride crucible with a conical-shaped bottom and then sealed in a quartz tube under a vacuum. The tube was placed in a vertical Bridgman furnace, heated to $1010^{\circ} \mathrm{C}$ at $100^{\circ} \mathrm{C} / \mathrm{h}$, and then held at this temperature for $24 \mathrm{~h}$. Crystal growth was carried out by lowering the tube at a rate of $3 \mathrm{~mm} / \mathrm{h}$. 

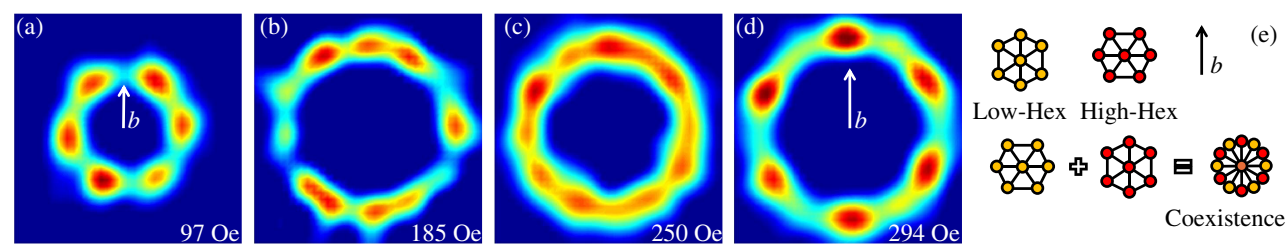

FIG. 1 (color online). (a)-(d) SANS diffraction patterns of CAS taken at $2 \mathrm{~K}$ in applied magnetic fields of 97, 185, 250, and 294 Oe, respectively. (e) Schematic diagram of the FLL patterns in real space (upper panel) and the corresponding diffraction patterns (lower panel).

SANS measurements were performed using the D22 instrument at the Institut Laue-Langevin, Grenoble, France. Additional anisotropy data were collected using the SANS I instrument at the Paul Scherrer Institut, Villigen, Switzerland [30]. The samples were mounted with the $c$ axis parallel to the neutron beam direction to access the Bragg peaks. To scan through the Bragg condition for a diffraction spot, a rocking curve was performed by tilting or rotating the sample, cryostat, and magnet together about a horizontal or vertical axis. For all measurements, the sample was cooled to base temperature in an applied magnetic field $H$ and the data collected while warming the sample in the same field. Background scattering was measured above $T_{c}$ and subtracted from the low-temperature data.

Data were collected in applied fields of 54 to 2000 Oe. Figures 1(a)-1(d) show typical diffraction patterns from the FLL of CAS measured at $2 \mathrm{~K}$ in fields of (a) 97, (b) 185, (c) 250, and (d) 294 Oe applied parallel to the $c$ axis. The only previous SANS measurements on $6 \mathrm{H}$-CAS suggested that the FLL was not perfectly hexagonal [31]. In our measurements, a perfectly hexagonal lattice was found for all applied fields. We find a well-defined FLL in an applied field of just 54 Oe. This is one of the lowest fields in which a FLL has been observed using the SANS technique. This field is also much smaller than the reported value of $H_{c 1}$ for $6 H$-CAS [7]. However, from magnetization versus field measurements, we estimate $H_{c 1}=50$ Oe. Just above $H_{c 1}$, the intervortex distance is several times larger than the penetration depth and any inhomogeneity may be expected to disrupt the FLL, leading to disordered vortex clusters.

In low fields [Fig. 1(a)], the Bragg peaks in the diffraction pattern, denoted here as Low-Hex, appear at $30^{\circ}$ to the $b$ axis of the crystal. With increasing field, a second hexagonal diffraction pattern appears oriented along the $b$ axis [see Figs. 1(b) and 1(c)]. This means that the FLL has now formed two domains with an angular separation of $30^{\circ}$. As the applied field is increased further, the FLL transforms into a single domain, with Bragg peaks oriented along the $b$ axis and referred to as High-Hex [Fig. 1(d)]. We do not observe any intermediate structures or any continuous change in the positions of the diffraction peaks during the reorientation process. These observations suggest that the transition between the High and Low-Hex phases is first-order in character. No further reorientations of the FLL were observed in applied fields of up to $2 \mathrm{kOe}$. An earlier SANS study of $6 \mathrm{H}$-CAS found no evidence for a FLL reorientation, as the measurements were not carried out in sufficiently low applied fields [31].

An $H-T$ phase diagram of CAS is shown in Fig. 2, indicating the regions in which we observe either a purely Low-Hex or a High-Hex phase separated by a shaded region in which the two FLL structures coexist. Figure 3 shows the variation of the integrated intensity of the Bragg spots for the High and Low-Hex states with applied magnetic field at $4 \mathrm{~K}$. A sudden change of intensity for the two states occurs through the narrow window of coexistence. In addition to the first-order nature of the transition, the coexistence of the two phases is due to a combination of shape demagnetization effects and pinning in the sample.

What drives the FLL reorientation in CAS? In $\mathrm{MgB}_{2}$, a $30^{\circ}$ reorientation in the FLL has been associated with the suppression of the smaller of the two superconducting gaps present in the material. However, the reorientation field $H_{r}$, for $\mathrm{MgB}_{2}$, is over $5 \mathrm{kOe}\left(H_{c 2} / H_{r} \approx 5\right)$, and the reorientation process is second-order [28]. The $30^{\circ}$ reorientation of the FLL reported here occurs in a field of only 200 Oe, which is a small fraction of $H_{c 2}=8 \mathrm{kOe}$ for this material, $\left(H_{c 2} / H_{r} \approx 40\right)$. For $6 H$-CAS, the experimental data suggest that, if two gaps are present, the ratio of their

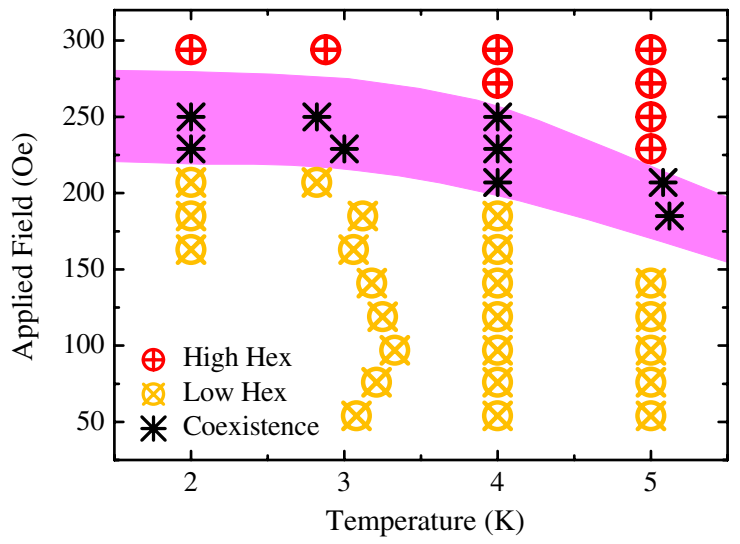

FIG. 2 (color online). $\quad H-T$ phase diagram of CAS indicating the temperatures and applied fields at which we observe either a High-Hex or a Low-Hex state for the FLL. A shaded region in which the two states coexist is also marked. 


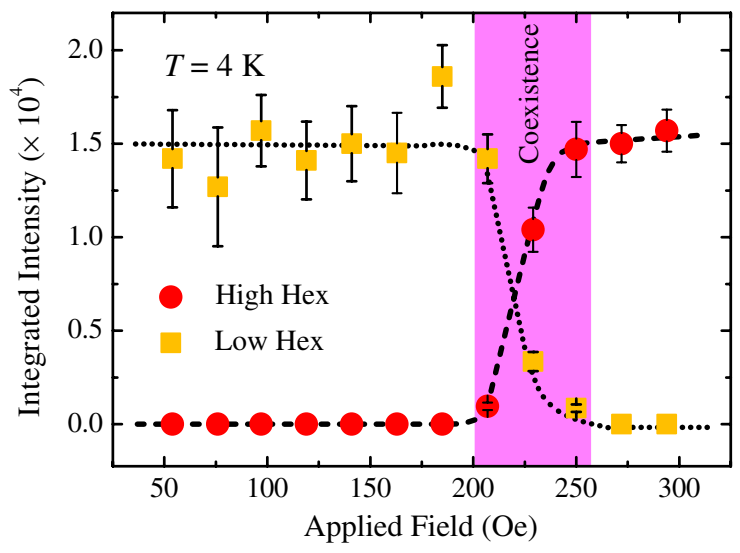

FIG. 3 (color online). Standard monitor normalized intensity of the Bragg peaks for the High-Hex and Low-Hex phases of CAS. The dotted and dashed lines are guides to the eye. The shading indicates the region in which the two FLL phases coexist.

magnitudes $\Delta_{c 1} / \Delta_{c 2} \leq 3.5[15,16]$. We conclude, therefore, that the reorientation of the FLL in CAS is not caused by the closing of the smaller of the two superconducting gaps.

In some ways, the FLL transition in CAS more closely resembles the (apparently) first-order $45^{\circ}$ reorientation between two rhombic FLL phases observed in Lu and Y borocarbide [21,32,33]. $H_{r}$ is 250 and 1500 Oe for Lu and Y borocarbide, respectively, with $\left(H_{c 2} / H_{r} \approx 50\right)$. Changes in the symmetry of the FLL and its orientation with respect to the high-symmetry directions in the crystal result from an anisotropy in the penetration depth and can be understood by considering nonlocal corrections to the London model $[21,32,34,35]$. When the intervortex distance is comparable to the penetration depth, the nonlocal effects play their part to align the vortices with the strong vortexvortex interactions.

Nonlocal effects are expected to be significant in a low- $\kappa$ superconductor like CAS. At the reorientation field $H_{r}=200 \mathrm{Oe}$, the intervortex distance in the hexagonal FLL is the same order of magnitude as the penetration depth. In addition, the morphology of the FLL and the $30^{\circ}$ reorientation reflects the underlying symmetry of the crystallographic lattice. These facts strongly suggest that nonlocal effects are driving the low-field reorientation of the FLL in CAS. To unambiguously establish this will require a calculation of the nonlocal corrections to a London model for a material with sixfold symmetry. This will be difficult, given the need for complicated Fermi surface averages.

Figure 4 shows the form factor $F$ at $2 \mathrm{~K}$, extracted from the integrated intensity of the Bragg spots forming the FLL in CAS. The form factor provides a measure of the amplitude of the field modulation inside a type-II superconductor due to the formation of a FLL [28]. According to the London model, for a conventional single-band supercon-

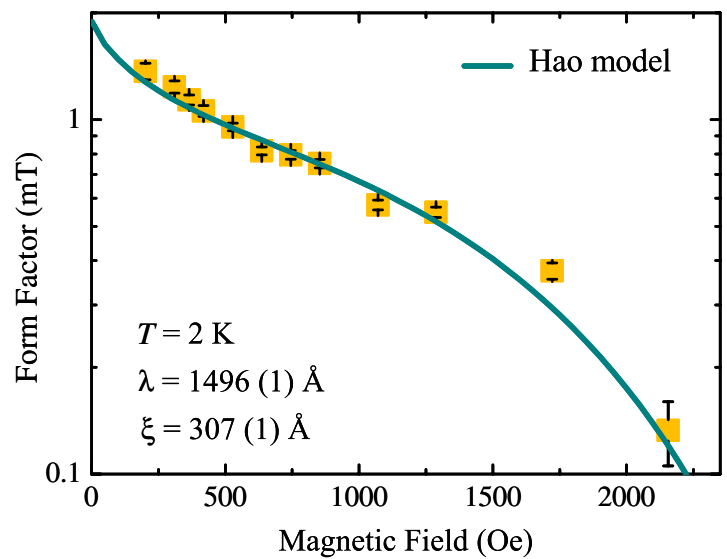

FIG. 4 (color online). Form factor $F$ of CAS plotted on a log scale. The solid line is a fit to the data using the Hao model described in the text and in Ref. [37].

ductor with a penetration depth and a coherence length that are independent of field, $F$ decreases exponentially with field [36]. However, for an anisotropic superconductor, an expression for $F$ has been calculated by Hao et al. [37] within the Ginzburg-Landau (GL) approximation.

$$
F=\frac{3^{1 / 4}}{2 \pi \sqrt{2}} \frac{\sqrt{\Phi_{0} B} f^{2} \xi_{v}}{\lambda^{2}} K_{1}\left(\frac{2 \pi \sqrt{2}}{3^{1 / 4}} \xi_{v} \sqrt{B / \Phi_{0}}\right)
$$

where

$$
\begin{aligned}
& \xi_{v}=\xi\left(\sqrt{2}-\frac{0.75}{\kappa}\right) \sqrt{\left(1+b^{4}\right)\left[1-2 b(1-b)^{2}\right]}, \\
& f^{2}=1-b^{4},
\end{aligned}
$$

$K_{n}(x)$ is a modified Bessel function of the $n$th order, $\Phi_{0}=$ $2.068 \times 10^{-15} \mathrm{~Wb}$ is the magnetic flux quantum, $\kappa=\lambda / \xi$ is the GL parameter, $B_{c 2}=\Phi_{0} /\left(2 \pi \xi^{2}\right)$ is the upper critical field, and $B=b B_{c 2}$ is the applied field [36,38]. The fit yields $\lambda=1496(1) \AA, \quad \xi=307(1) \AA$, and $\kappa=4.9(1)$. This $\kappa$ is consistent with the value of 5.2 reported by Imai et al. [7]. $\xi$ is $50 \%$ larger than the value extracted from $H_{c 2}$ measurements on the same sample. In a study of $\mathrm{MgB}_{2}$, the increase in $F$ at low field was attributed to a change in the superfluid density [28]. As shown here, such a conclusion is not required for CAS.

The penetration depth anisotropy $\gamma_{\lambda}$ can be extracted by rotating the applied magnetic field away from the $c$ axis
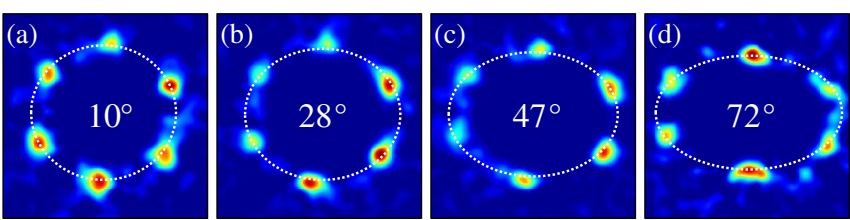

FIG. 5 (color online). (a-d) Anisotropic FLL of CAS at $1.5 \mathrm{~K}$ and a field of $3 \mathrm{kOe}$ applied at $10^{\circ}, 28^{\circ}, 47^{\circ}$, and $72^{\circ}$, respectively, to the $c$ axis of the crystal. 


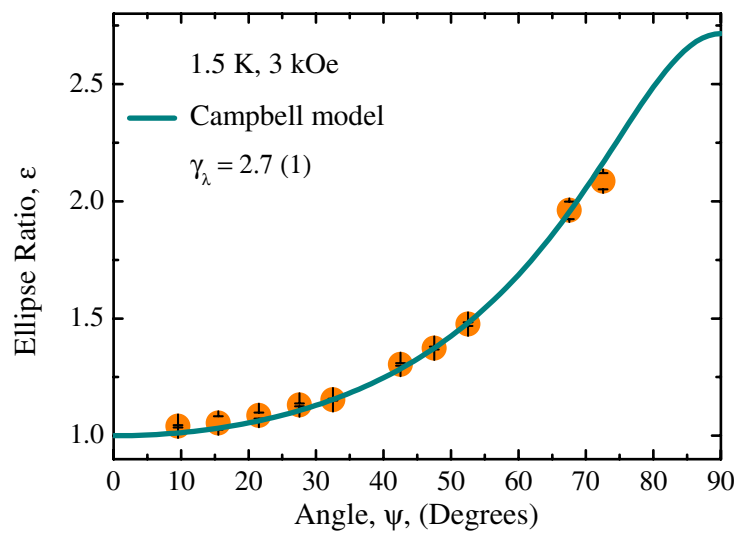

FIG. 6 (color online). Ellipse ratio $\epsilon$, as a function of the angle $\psi$ at $1.5 \mathrm{~K}$ and applied field of $3 \mathrm{kOe}$ for CAS. The solid line is fit to the data using the Campbell model and yields $\gamma_{\lambda}=2.7(1)$ at $\psi=90^{\circ}$.

and measuring the ratio of the major to minor axes of the ellipse $(\epsilon)$ connecting the Bragg peaks. Figures 5(a)-5(d) show the diffraction patterns of CAS taken at $1.5 \mathrm{~K}$ in a field of $3 \mathrm{kOe}$ applied at $10^{\circ}, 28^{\circ}, 47^{\circ}$, and $72^{\circ}$, respectively, to the $c$ axis. As the angle between the applied field and the $c$ axis increases, the diffraction pattern is distorted towards an elliptical shape, since the screening currents circulating around a vortex must cross the basal plane. Campbell et al. [39] studied the structure of a vortex lattice in anisotropic, uniaxial superconductors, for magnetic fields applied at an angle $\psi$ to the principal axis. According to their model based on the London approach, $\epsilon$ is related to $\gamma_{\lambda}$ in the following way,

$$
\epsilon^{2}=\frac{\gamma_{\lambda}^{2}}{\sin ^{2} \psi+\gamma_{\lambda}^{2} \cos ^{2} \psi}
$$

Figure 6 shows the variation of $\epsilon$ as a function of $\psi$ for CAS measured at $1.5 \mathrm{~K}$ in a field of $3 \mathrm{kOe}$. A fit to the data using Eq. (3) is indicated by the solid line, yielding an anisotropy $\gamma_{\lambda}=2.7(1)$. The value of $\gamma_{\lambda}$ is in excellent agreement with previous values of $\gamma_{\xi}$ determined from magnetic and transport measurements [7,8] and slightly larger than the value of 2 obtained by Kuroiwa et al. [31] from SANS measurements.

Close to $T_{c}$, the anisotropic GL equations for a clean superconductor with an arbitrary gap anisotropy yield $\gamma_{\lambda}=\gamma_{\xi}$. At lower $T$, however, these two quantities may both depend on $T$ and are not necessarily the same. For example, in the case of $\mathrm{MgB}_{2}$, calculations for a weakly coupled two-band anisotropic superconductor showed that $\gamma_{\lambda}(T)$ and $\gamma_{\xi}(T)$ are increasing and decreasing functions of $T$, respectively [40,41]. In CAS, the equality of $\gamma_{\lambda}$ and $\gamma_{\xi}$ at $1.5 \mathrm{~K}$ may reflect the fact that the morphology of FLL is established at higher $T$ and then gets pinned as $T$ is reduced. Alternatively, it may be indicative of a more isotropic character for the Fermi surface in this material.
This work was supported by the Engineering and Physical Sciences Research Council (EPSRC). Some of the equipment used in this research was obtained through the Science City Advanced Materials project: Creating and Characterizing Next Generation Advanced Materials project, with support from Advantage West Midlands (AWM) and part funded by the European Regional Development Fund (ERDF). D. S. K thanks the Science City Research Alliance and the HEFCE Strategic Development Fund for financial support. P. K. B would like to thank the Midlands Physics Alliance Graduate School (MPAGS) for financial support. The authors would like to thank V. Kogan for valuable discussions and interest in this work.

*P.K.Biswas@warwick.ac.uk

[1] M. Imai et al., Appl. Phys. Lett. 80, 1019 (2002).

[2] B. Lorenz et al., Physica (Amsterdam) 383C, 191 (2002).

[3] B. Lorenz et al., Phys. Rev. B 68, 014512 (2003).

[4] H. Sagayama et al., J. Phys. Soc. Jpn. 75, 043713 (2006).

[5] J. Nagamatsu et al., Nature (London) 410, 63 (2001).

[6] S. Kuroiwa et al., Phys. Rev. B 74, 014517 (2006).

[7] M. Imai et al., Phys. Rev. B 68, 064512 (2003).

[8] A. K. Ghosh, M. Tokunaga, and T. Tamegai, Phys. Rev. B 68, 054507 (2003).

[9] S. Kuroiwa et al., J. Phys. Chem. Solids 68, 2124 (2007).

[10] L. Boeri et al., Phys. Rev. B 77, 144502 (2008).

[11] I. R. Shein, N. I. Medvedeva, and A. L. Ivanovskii, J. Phys. Condens. Matter 15, L541 (2003).

[12] G. Q. Huang et al., Phys. Rev. B 69, 064509 (2004).

[13] I. I. Mazin and D. A. Papaconstantopoulos, Phys. Rev. B 69, 180512 (2004).

[14] S. Kuroiwa et al., J. Phys. Soc. Jpn. 76, 113705 (2007).

[15] S. Tsuda et al., Phys. Rev. B 69, 100506 (2004).

[16] S. Lupi et al., Phys. Rev. B 77, 054510 (2008).

[17] R. Prozorov et al., Phys. Rev. B 73, 184523 (2006).

[18] S. Kuroiwa et al., Phys. Rev. B 76, 104508 (2007).

[19] R. Cubitt et al., Nature (London) 365, 407 (1993).

[20] Y. De Wilde et al., Phys. Rev. Lett. 78, 4273 (1997).

[21] D. M. Paul et al., Phys. Rev. Lett. 80, 1517 (1998).

[22] V. G. Kogan et al., Phys. Rev. Lett. 79, 741 (1997).

[23] A. Gurevich and V. G. Kogan, Phys. Rev. Lett. 87, 177009 (2001).

[24] N. Nakai et al., Phys. Rev. Lett. 89, 237004 (2002).

[25] S. J. Levett, C. D. Dewhurst, and D. M. Paul, Phys. Rev. B 66, 014515 (2002).

[26] V. G. Kogan et al., Phys. Rev. B 55, R8693 (1997).

[27] K. Park and D. A. Huse, Phys. Rev. B 58, 9427 (1998).

[28] R. Cubitt et al., Phys. Rev. Lett. 91, 047002 (2003).

[29] See Supplemental Material at http://link.aps.org/ supplemental/10.1103/PhysRevLett.108.077001 for an $\mathrm{x}$-ray diffraction study of the single crystal used in this work which confirms that the sample has the $6 H$ phase of CAS.

[30] D22 was configured in a high-resolution mode with a mean wavelength of $14 \AA$ and a wavelength spread of $10 \%$, collimation of $17.6 \mathrm{~m}$, and an area detector at a 
distance of $17.6 \mathrm{~m}$ to the sample. An additional circular aperture of diameter $20 \mathrm{~mm}$ was also used before the sample to better define the scattered beam at the detector and to equalize the horizontal and vertical divergences. At SANS I, incident neutrons of various wavelengths between 5-10 $\AA$ were selected with a wavelength spread of $10 \%$ and collimated over a distance of 8 to $18 \mathrm{~m}$ before the sample. Diffracted neutrons were collected with a position-sensitive two-dimensional multidetector located 13-20 $\mathrm{m}$ after the sample.

[31] S. Kuroiwa et al., J. Phys. Chem. Solids 68, 2129 (2007).

[32] C. D. Dewhurst, S. J. Levett, and D. M. Paul, Phys. Rev. B 72, 014542 (2005).
[33] L. Ya. Vinnikov et al., Phys. Rev. B 64, 220508 (2001).

[34] M. Yethiraj et al., Phys. Rev. B 58, R14767 (1998).

[35] V. G. Kogan, P. Miranovic, and D. M. Paul, Vortex Lattice Transitions, edited by C.A.R. Sa de Melo (World Scientific, Singapore, 1998), p. 127.

[36] A. Yaouanc, P. Dalmas de Réotier, and E. H. Brandt, Phys. Rev. B 55, 11107 (1997).

[37] Z. Hao et al., Phys. Rev. B 43, 2844 (1991).

[38] E. H. Brandt, Phys. Rev. B 37, 2349 (1988).

[39] L. J. Campbell, M. M. Doria, and V. G. Kogan, Phys. Rev. B 38, 2439 (1988).

[40] V. G. Kogan, Phys. Rev. B 66, 020509 (2002).

[41] P. Miranović, K. Machida, and V. G. Kogan, J. Phys. Soc. Jpn. 72, 221 (2003). 